\title{
Erratum to: High-Resolution Structure of a Membrane Protein Transferred from Amphipol to a Lipidic Mesophase
}

\author{
V. Polovinkin 1,2,3,4 $\cdot$ I. Gushchin ${ }^{1,2,3,4} \cdot$ M. Sintsov ${ }^{4} \cdot$ E. Round $^{5} \cdot$ T. Balandin ${ }^{5}$. \\ P. Chervakov ${ }^{5}$. V. Shevchenko ${ }^{5} \cdot$ P. Utrobin ${ }^{1,2,3}$ - A. Popov ${ }^{6}$ V. Borshchevskiy ${ }^{4,5}$. \\ A. Mishin ${ }^{4}$ A. Kuklin ${ }^{7}$ D. Willbold ${ }^{5,8} \cdot$ V. Chupin ${ }^{4}$ J.-L. Popot ${ }^{9}$ V. Gordeliy 1,2,3,4,5
}

Published online: 22 February 2017

(C) Springer Science+Business Media New York 2017

\section{Erratum to: J Membrane Biol (2014) 247:997-1004 \\ DOI 10.1007/s00232-014-9700-x}

The original version of this article unfortunately contained an error. The correct surname of the co-author is Shevchenko, it originally appeared incorrectly as Schevchenko.

The online version of the original article can be found under doi:10.1007/s00232-014-9700-x.

V. Gordeliy

valentin.gordeliy@ibs.fr

1 Univ. Grenoble Alpes, IBS, 38044 Grenoble, France

2 CNRS, IBS, 38044 Grenoble, France

3 CEA, IBS, 38044 Grenoble, France

4 Laboratory for Advanced Studies of Membrane Proteins, Moscow Institute of Physics and Technology, 141700 Dolgoprudny, Moscow Region, Russia

5 Institute of Complex Systems (ICS), ICS-6: Structural Biochemistry, Research Centre Juelich, 52425 Juelich, Germany

6 European Synchrotron Radiation Facility, 38027 Grenoble, France
7 Frank Laboratory of Neutron Physics, Joint Institute for Nuclear Research, 141980 Dubna, Moscow Region, Russia

8 Institut für Physikalische Biologie, Heinrich-HeineUniversität Düsseldorf, 40225 Düsseldorf, Germany

9 Laboratoire de Physico-Chimie Moléculaire des Membranes Biologiques, UMR 7099, Institut de Biologie Physico-Chimique (CNRS FRC 550), Centre National de la Recherche Scientifique and Université Paris-7, 13 rue Pierre et Marie Curie, 75005 Paris, France 